
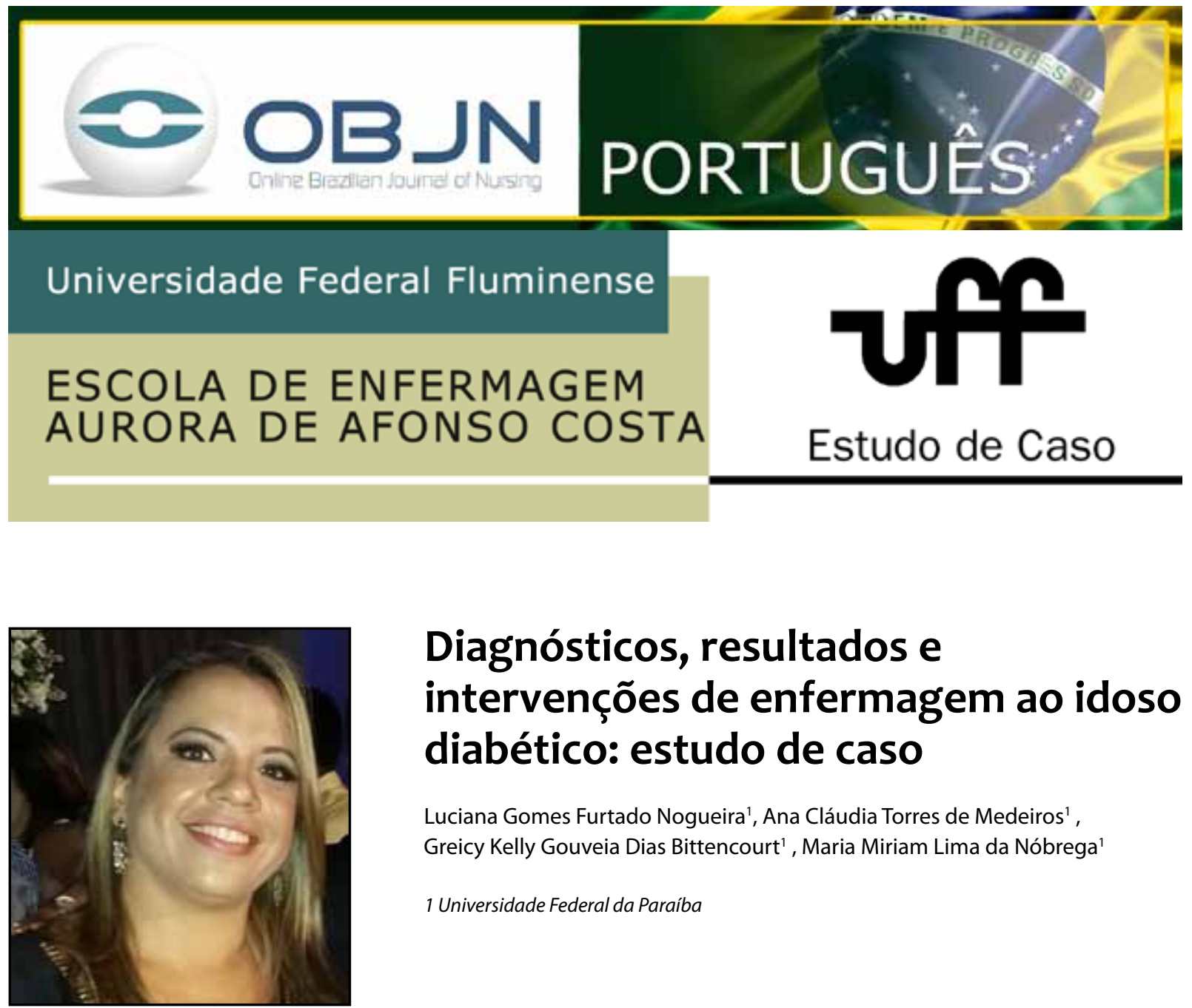

\title{
Diagnósticos, resultados e intervenções de enfermagem ao idoso diabético: estudo de caso
}

\author{
Luciana Gomes Furtado Nogueira', Ana Cláudia Torres de Medeiros , \\ Greicy Kelly Gouveia Dias Bittencourt ${ }^{1}$, Maria Miriam Lima da Nóbrega \\ 1 Universidade Federal da Paraíba
}

\section{RESUMO}

Objetivo: identificar diagnósticos, resultados e intervenções de enfermagem a um idoso portador de diabetes mellitus utilizando a Classificação Internacional para a Prática da Enfermagem (CIPE') 2011. Método: estudo de caso descrevendo a assistência de enfermagem com identificação de diagnósticos, resultados e intervenções de enfermagem utilizando a CIPE 2011 . Resultados: foram priorizados nove diagnósticos: dispneia aos mínimos esforços; volume de líquidos excessivo (edema); déficit de autocuidado parcial para alimentar-se; déficit de autocuidado total para banho, vestir-se, higiene; ferida diabética no membro inferior esquerdo; integridade da pele prejudicada no abdome; dor intensa em membros inferiores; falta de adesão ao regime terapêutico e falta de conhecimento sobre a doença. Discussão: a maioria dos diagnósticos de enfermagem verificados está relacionada intimamente às complicações crônicas do diabetes mellitus, acrescidas das alterações fisiológicas do envelhecimento.

Descritores: Diabetes Mellitus; Idoso; Assistência ao Paciente; Processos de Enfermagem; Diagnóstico de Enfermagem. 


\section{INTRODUÇÃO}

Nas últimas décadas, o Brasil tem apresentado um processo de envelhecimento de sua população de forma acelerada abrindo perspectivas de acordo com os últimos censos demográficos para pirâmides etárias semelhantes a de países europeus, sendo essas transformações responsáveis em colocar desafios para todos os setores ${ }^{(1)}$.

Dentre as implicações advindas do enveIhecimento populacional, destaca-se o aumento da prevalência e doenças crônicas (circulatórias, pulmonares, osteoarticulares, alterações neuropsiquiátricas e diabetes mellitus), constituindo um importante problema de saúde pública decorrente o seu elevado impacto de morbidade, mortalidade, redução da qualidade de vida e de custos da assistência de saúde ${ }^{(2)}$.

Dentre as doenças crônicas destaca-se a diabetes mellitus (DM), definida como um grupo de doenças metabólicas caracterizadas por hiperglicemia resultante de alterações na secreção e/ou ação da insulina. É classificada fisiopatologicamente em quatro grandes classes clínicas: DM tipo 1, DM tipo 2, outros tipos de diabetes e DM gestacional; podendo durante sua evolução, na dependência do controle metabólico, advir complicações agudas e crônicas, micro e macrovasculares, como as doenças coronarianas, retinopatia, nefropatia, insuficiência vascular periférica, neuropatia periférica, neuropatia autonômica, entre outros ${ }^{(3,4)}$.

Os recentes dados epidemiológicos têm evidenciado o DM tipo 2 como um dos maiores incrementos de prevalência, adquirindo características epidêmicas, especialmente na população idosa, alterando a atual prevalência de $20 \%$ para $50 \%$ nas próximas duas décadas. A razão para essa prevalência entre idosos é decorrente de processos fisiopatológicos, como a maior deposição de beta mieloide, ocasionando disfunção da célula beta com menor produção de insulina e diminuição da massa magra, aumento da massa gorda na região abdominal e diminuição da atividade física, provocando a resistência insulínica ${ }^{(2)}$.

Além da maior prevalência de acometimento de doenças crônicas, os idosos apresentam uma condição particular de agravamento das condições de saúde, que é o desenvolvimento das Síndromes Geriátricas (SG) - alterações associadas ao envelhecimento orgânico e da ação deletéria acumulada nos diferentes sistemas decorrentes das doenças crônicas, como incontinência urinária, instabilidade e maior risco de quedas, iatrogenia secundária a polifarmácia em virtude das múltiplas comorbidades que acompanham o DM, depressão e demência, tornando o seu tratamento mais complexo ${ }^{(2)}$.

Os portadores de DM, em particular os idosos, necessitam de um acompanhamento sistemático por uma equipe multiprofissional de saúde que ofereça as ferramentas necessárias para o manejo da doença com vistas a alcançar níveis normais de glicose sanguínea evitando complicações agudas e crônicas, com uma adequação satisfatória ao estilo de vida(4).

Nessa perspectiva, como membro da equipe multiprofissional o enfermeiro deve estar capacitado para o atendimento integral a essa clientela, utilizando o processo de enfermagem, considerado uma variação do raciocínio científico que ajuda o enfermeiro a organizar, sistematizar e conceituar a prática de enfermagem ${ }^{(5)}$.

Evidenciam-se estudos voltados para o cuidado ao idoso ou ao diabético ${ }^{(4,5)}$, porém há necessidade de pesquisas sobre o idoso diabético, ressaltando-se a importância de se investigar como se dá a assistência de enfermagem ao idoso portador de DM a partir da identificação de diagnósticos, resultados e intervenções de enfermagem. Desse modo, pretende-se contribuir para o avanço do conhecimento de 
enfermagem nessa área, além de colaborar com o desenvolvimento de uma linguagem específica da enfermagem ao construir tais elementos da prática com um sistema de classificação de âmbito internacional como a Classificação Internacional para a Prática de Enfermagem - CIPE`.

Visando ao conhecimento de evidências científicas para o cuidado integral a essa clientela, desenvolveu-se este estudo com o objetivo de identificar diagnósticos, resultados e intervenções de enfermagem a um idoso portador de diabetes mellitus utilizando a CIPE 2011.

\section{MÉTODO}

Trata-se de um estudo de caso com um idoso portador de diabetes mellitus tipo 2, realizado em um setor de Clínica Médica de um hospital público de João Pessoa/PB, no período de fevereiro a março de 2012, respeitando-se os aspectos éticos da Resolução 466/12, sobre pesquisas $^{(6)}$ que envolvem seres humanos. Ressalta-se que o estudo encontra-se vinculado a um projeto de pesquisa sobre sistematização da assistência de enfermagem com aprovação sob o protocolo n 054/2007.

Para desenvolver o estudo de caso, aplicou-se o processo de enfermagem em etapas dinâmicas: coleta de dados, diagnóstico, planejamento e avaliação da assistência de enfermagem. Para a coleta de dados, utilizou-se o histórico de enfermagem ${ }^{(7)}$ do respectivo setor, o qual é embasado na Teoria das Necessidades Humanas Básicas de Horta, sendo disposto por necessidades. Utilizou-se a CIPE $^{\circ} 2011$ para identificação dos diagnósticos, resultados e intervenções de enfermagem visando ao cuidado do idoso portador de DM tipo 2.

Para elucidar o raciocínio diagnóstico, recorreu-se à construção de um mapa conceitual a fim de demonstrar a identificação, relação e agrupamento de conceitos constituintes dos diagnósticos, resultados de enfermagem. Para tanto, utilizou-se o CMap Tools em sua versão 5.03, que é um software desenvolvido e distribuído gratuitamente pelo Institute for Human Machine Cognition da University of West Florida ${ }^{(8)}$, o qual permite o usuário construir, navegar, compartilhar e criticar modelos de conhecimento representados com mapas conceituais. Assim, identificaram-se conceitos considerados neste estudo como sinais, sintomas, fatores de risco e condições de vida, que foram organizados hierarquicamente, apresentando a relação e o agrupamento entre eles por meio do mapa conceitual.

Dessa forma, foi possível identificá-los, ordená-los e agrupá-los observando suas relações, para posterior construção das afirmativas de diagnósticos e resultados de enfermagem. Ressalta-se que, neste estudo, utilizou-se a expressão "diagnóstico e resultado de enfermagem" para denominar esses dois elementos da prática de enfermagem, tendo em vista que se utilizam os mesmos eixos da $\mathrm{CIPE}^{\circ}$ foco e julgamento na sua construção, e o que determina a diferença entre os mesmos é a avaliação do enfermeiro sobre se é uma decisão a respeito do estado do cliente, problemas e ou necessidades (diagnóstico) ou se é a resposta dada depois da implementação das intervenções (resultado).

Com base na identificação dos diagnósticos e resultados, foi possível planejar as intervenções de enfermagem realizando a avaliação dos resultados alcançados.

\section{RESULTADOS}

Nesta sessão, apresenta-se um caso clínico de um idoso portador de diabetes mellitus para identificar sinais, sintomas, respostas humanas para posterior construção de diagnósticos/ 
resultados e intervenções de enfermagem com base na CIPE 2011.

\section{Caso clínico}

AJA, 67 anos, moreno, sexo masculino, $2^{\circ}$ grau incompleto, aposentado, casado, $53 \mathrm{~kg}$, 1,60m de altura, natural de João Pessoa, católico, procedente de sua residência, em cadeira de rodas. Diagnóstico médico de insuficiência cardíaca congestiva descompensada, doença arterial coronariana, diabetes mellitus descompensada, nefropatia diabética, ferida diabética no membro inferior esquerdo. Queixava-se de cansaço, dispneia e edema (generalizado). Necessidades/Autocuidado: restrito ao leito, necessitando de ajuda para banhar-se, higienizar-se e vestir-se. Apresentava sono e repouso prejudicados devido à dispneia e tosse, aceitando cinco refeições diárias, com boa ingesta hídrica, recebendo ajuda dos filhos para o cuidado. Apresentava-se pouco informado sobre seu problema de saúde. Exame físico: estado geral grave, consciente, desorientação espacial leve, difícil comunicação, face de sofrimento, gemente, restrito ao leito, em anasarca, olho esquerdo com prótese ocular, acuidade auditiva diminuída, presença de acesso venoso central na veia jugular esquerda, dispneico, uso de oxigênio suplementar por cateter nasal, tosse seca intermitente, atividade cardíaca regular, abdome globoso, presença de lesões eritemato-descamativa exsudativa, ruídos hidroaéreos presentes, diurese colúrica por sonda vesical de demora apresentando albuminúria, eliminações fecais preservadas, parestesia de membros inferiores, pulsos periféricos diminuídos, referindo dor em membros inferiores (MMII) (claudicação intermitente), fissura no hálux esquerdo, lesão vésico-bolhosa supurativa em membro inferior esquerdo. Sinais vitais - T: $36^{\circ} \mathrm{C} ; \mathrm{P}: 86$ bpm; R:19 irpm; PA: 120x80mmHg.
Mapa conceitual - apresentação do raciocínio diagnóstico

Diante do estudo de caso apresentado, foram evidenciados indicadores empíricos que subsidiaram o processo de raciocínio clinico permitindo a identificação de 19 diagnósticos de enfermagem com base no quadro clínico do paciente com complicações crônicas do DM associado ao processo de envelhecimento. Foram priorizados nove diagnósticos de enfermagem destacados em negrito, considerando-se o que realmente seria foco de atenção da prática de enfermagem que podem ser visualizados no Mapa Conceitual (Figura 1).

Com base na identificação dos diagnósticos conforme demonstrado na Figura 1, apresenta-se a Figura 2 para ilustrar os diagnósticos, resultados e intervenções de enfermagem planejadas para o idoso portador de diabetes mellitus e a avaliação da assistência de enfermagem.

Nessa situação, a partir das intervenções implementadas, o paciente foi mantido em decúbito elevado, consciente, em uso de máscara de venturi, monitorado com saturação de 98\%. O edema apresentou discreta redução, porém foi possível proteger a pele de possíveis lesões com utilização de coxins e mudança de decúbito. Apresentou uma boa aceitação alimentar com ajuda de acompanhante e equipe de enfermagem. Manteve-se com a higiene oral e corporal preservadas, sem presença de úlcera por pressão, mas ainda dependente da equipe de enfermagem decorrente do quadro clínico prejudicado. A ferida diabética do membro inferior esquerdo foi debridada em bloco cirúrgico e manteve-se em estágio de granulação; apresentou melhora da lesão do abdome a partir dos curativos realizados. A dor foi minimizada a partir de medidas de conforto como aquecimento dos pés com meias e posicionamento do paciente associado com a terapia medicamentosa. 
Figura 1 - Diagnósticos de enfermagem prioritários em idoso portador de diabetes mellitus com base nas necessidades humanas básicas afetadas. João Pessoa, 2012.

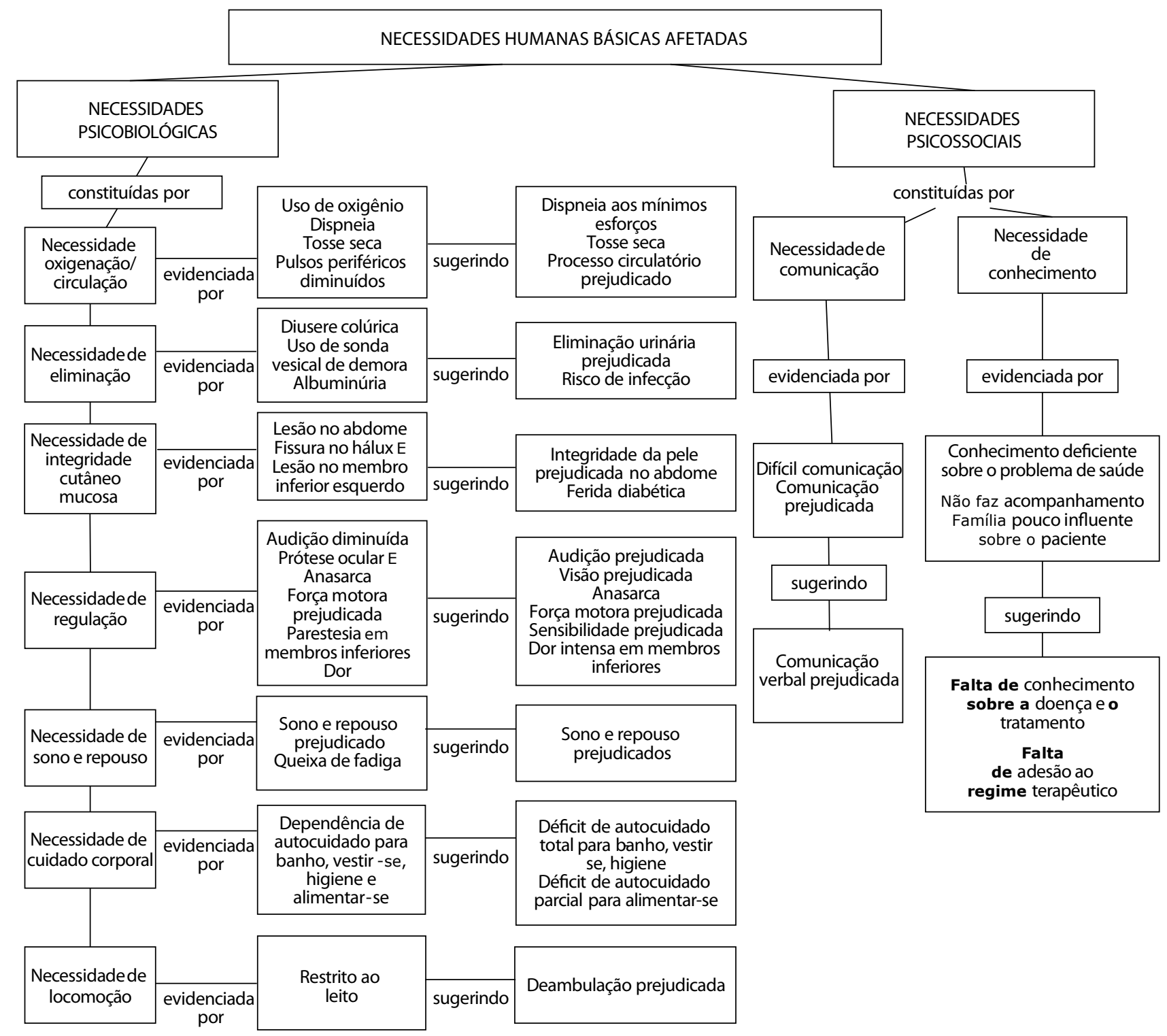

As intervenções relacionadas à educação em saúde foram implementadas junto aos familiares, responsáveis pelo cuidado do idoso, demonstrando uma maior compreensão em relação à importância de seguir o regime terapêutico.

\section{DISCUSSÃO}

Neste estudo, verifica-se que dos 19 diagnósticos de enfermagem identificados, a maioria está relacionado intimamente às complicações crônicas do DM, acrescidas das alterações fisiológicas do envelhecimento, evidenciando uma sucessão de acontecimentos durante a evolução da doença pela falta de prevenção de fatores de risco. Isso possibilita o repensar sobre a atenção integral que deve ser disponibilizada para tal clientela desde a Atenção Primária, para que sejam minimizados casos semelhantes a este.

Dessa forma, serão discutidos os nove diagnósticos de enfermagem que constituíram o planejamento da assistência, ressaltando-se 
Figura 2 - Planejamento da assistência de enfermagem a um idoso portador de diabetes mellitus. João Pessoa, 2012.

\begin{tabular}{|c|c|c|}
\hline $\begin{array}{l}\text { Diagnósticos de enferma- } \\
\text { gem }\end{array}$ & $\begin{array}{l}\text { Resultados de enferma- } \\
\text { gem }\end{array}$ & Intervenções de enfermagem \\
\hline $\begin{array}{l}\text { Dispneia aos mínimos } \\
\text { esforços }\end{array}$ & Dispneia melhorada & $\begin{array}{l}\text { Avaliar condições hemodinâmicas (SPO2, FC, FR, nível de } \\
\text { consciência); manter a cabeceira elevada; monitorar padrão } \\
\text { respiratório; implementar e monitorar terapia de oxigênio. }\end{array}$ \\
\hline $\begin{array}{l}\text { Volume de líquidos exces- } \\
\text { sivo }\end{array}$ & $\begin{array}{l}\text { Volume de líquidos dimi- } \\
\text { nuído }\end{array}$ & $\begin{array}{l}\text { Avaliar as condições de pele e a perfusão; proteger a pele } \\
\text { edemaciada de lesões e oferecer apoio a áreas edemaciadas } \\
\text { quando necessário; avaliar e registrar a evolução do edema. }\end{array}$ \\
\hline $\begin{array}{l}\text { Déficit de autocuidado } \\
\text { parcial para alimentar-se }\end{array}$ & $\begin{array}{l}\text { Déficit de autocuidado me- } \\
\text { Ihorado para alimentar-se }\end{array}$ & $\begin{array}{l}\text { Auxiliar o paciente a adotar um melhor posicionamento para } \\
\text { se alimentar; auxiliar na alimentação. }\end{array}$ \\
\hline $\begin{array}{l}\text { Déficit de autocuidado } \\
\text { total para banho,vestir-se, } \\
\text { higiene }\end{array}$ & $\begin{array}{l}\text { Déficit de autocuidado } \\
\text { parcial para banho, vestir- } \\
\text {-se, higiene }\end{array}$ & $\begin{array}{l}\text { Realizar banho no leito; realizar trocas de roupas após o ba- } \\
\text { nho; trocar roupas de cama diariamente; observar condições } \\
\text { da pele durante banho e ou higiene; auxiliar na escovação dos } \\
\text { dentes e no enxague da boca, conforme condição do cliente. }\end{array}$ \\
\hline $\begin{array}{l}\text { Ferida diabética no mem- } \\
\text { bro inferior esquerdo }\end{array}$ & $\begin{array}{l}\text { Ferida diabética sem sinais } \\
\text { flogísticos }\end{array}$ & $\begin{array}{l}\text { Cuidar do local da ferida (limpeza, curativo e avaliação diária); } \\
\text { ensinar o paciente e família sobre o cuidado da ferida e cica- } \\
\text { trização. }\end{array}$ \\
\hline $\begin{array}{l}\text { Integridade da pele preju- } \\
\text { dicada no abdome }\end{array}$ & $\begin{array}{l}\text { Integridade da pele melho- } \\
\text { rada no abdome }\end{array}$ & $\begin{array}{l}\text { Avaliar a região afetada quanto às características da lesão; } \\
\text { realizar curativo diário ou quando necessário. }\end{array}$ \\
\hline $\begin{array}{l}\text { Dor intensa em membros } \\
\text { inferiores }\end{array}$ & $\begin{array}{l}\text { Dor melhorada em mem- } \\
\text { bros inferiores }\end{array}$ & $\begin{array}{l}\text { Avaliar e descrever as características da dor; promover confor- } \\
\text { to e medidas que ajudem na diminuição da dor. }\end{array}$ \\
\hline $\begin{array}{l}\text { Falta de adesão ao regime } \\
\text { terapêutico }\end{array}$ & $\begin{array}{l}\text { Adesão ao regime terapêu- } \\
\text { tico melhorado }\end{array}$ & $\begin{array}{l}\text { Orientar de acordo com o nível social as diretrizes do regime } \\
\text { terapêutico (atividade, alimentação saudável, monitorização } \\
\text { de glicemia, medicação, resolução de problemas, enfrenta- } \\
\text { mento saudável, redução de riscos). }\end{array}$ \\
\hline $\begin{array}{l}\text { Falta de conhecimento } \\
\text { sobre a doença }\end{array}$ & $\begin{array}{l}\text { Conhecimento adequado } \\
\text { sobre a doença }\end{array}$ & $\begin{array}{l}\text { Adaptar orientações segundo grau de compreensão dos fami- } \\
\text { liares; ensinar aos familiares a respeito da doença e tratamen- } \\
\text { to; orientar a respeito de suas limitações e a importância de } \\
\text { adesão ao regime terapêutico. }\end{array}$ \\
\hline
\end{tabular}

Fonte: autoria própria

que a assistência de enfermagem, neste caso, foi voltada para a minimização de complicações.

Em relação ao diagnóstico de enfermagem

Dispneia aos mínimos esforços, ocorreu a permanência do diagnóstico no decorrer da hospitalização, produzindo-Ihe desconfortos, que gerou a necessidade de oxigênio suplementar, impedindo-Ihe da preservação do sono e repouso e mantendo-o restrito ao leito. É definida pelo $\mathrm{CIE}^{(9)}$ como "movimento forçado de ar para dentro e fora dos pulmões, encurtamento da respiração associado à insuficiência de oxigênio no sangue circulante, sensação de desconforto e ansiedade". Neste caso está associada às complicações macrovasculares do
DM como a Doença Arterial Coronariana que provoca a insuficiência cardíaca reduzindo a função ventricular desencadeando congestão pulmonar, sendo caracterizada por falta de ar, agravando-se principalmente à noite, além de tosse e edema periférico.

A Doença Arterial Coronariana é considerada a complicação de maior comorbidade por estar associada a maior ocorrência de infarto agudo do miocárdio, óbitos e necessidade de revascularização miocárdica e à isquemia silenciosa $^{(3)}$. No idoso, o risco de desenvolvimento dessas complicações é bem evidente devido à prevalência que se tem de se desenvolver a hipertensão arterial, considerado um dos fatores 
de risco para as complicações macrovascula$\operatorname{res}^{(10)}$.

Esse diagnóstico de enfermagem é observado em idosos portadores de DM já acometidos com complicações, sendo a assistência de enfermagem, nessas situações, inserida na Atenção Terciária, tendo como foco a redução de complicações e a reabilitação.

Quanto ao diagnóstico de enfermagem Volume de líquidos excessivo, instalado no paciente de forma progressiva, demandou intervenções invasivas como acesso venoso central e sonda vesical de demora decorrente da dificuldade de acesso venoso periférico e edema peniano, requerendo da equipe de enfermagem uma monitorização diária devido ao risco de rompimento da pele.

Tal quadro de retenção hídrica é resultado de complicações crônicas instaladas no idoso diabético, deste estudo, como a nefropatia diabética e a doença arterial coronariana. A nefropatia diabética consiste em uma principal causa da insuficiência renal crônica, tendo seu início caracterizado por pequenas quantidades de albumina na urina, denominado de albuminúria e em estágios avançados macroalbuminúria, proteinúria ou nefropatia clínica ${ }^{(3)}$.

Como forma de se evitar a conversão da microalbuminúria em macroalbuminúria, destaca-se a importância de se avaliar anualmente a urina por meio do teste urinário para a microalbuminúria além de exames para se avaliar os níveis séricos de urina e creatinina. Adicionado a estas medidas, também são apontadas o controle da hipertensão arterial, prevenção ou tratamento das infecções do trato urinário, prevenção das substâncias nefrotóxicas, dieta hipossódica e hipoproteica(10).

Conviver com uma doença crônica de saúde pode acarretar situações relacionadas ao déficit de autocuidado. No idoso, em estudo, o diagnóstico de enfermagem Déficit de auto- cuidado foi avaliado como total nas atividades de banho, higiene e vestir-se e como Déficit de autocuidado parcial na atividade de alimentação, devido ao quadro clínico em que o paciente se encontrava. No entanto, era perceptível o quanto a situação de dependência o incomodava devido sua independência na realização dessas atividades.

Contudo, devido ao quadro de desorientação leve que o idoso se encontrava todas as intervenções de enfermagem relacionadas ao autocuidado foram partilhadas com os familiares que o acompanhavam visando o desenvolvimento de habilidades para o cuidado domiciliar.

O enfermeiro pode planejar práticas de autocuidado para o idoso com DM de acordo com as reais necessidades podendo ser alteradas desde que contribua positivamente sobre seu comportamento, mediante mudanças no estilo de vida, refletindo na qualidade de vida ${ }^{(11)}$. Embora o empenho pelo controle rigoroso dos níveis glicêmicos possa não ser seguro ou apropriado diante do risco de hipoglicemia, deve ser evitada a hiperglicemia prolongada.

Dessa forma, para esse tipo de cliente, torna-se importante a elaboração de um plano individual e efetivo pautado no contexto de vida integrando os familiares, uma vez que, existem algumas barreiras para o aprendizado e autocuidado como visão diminuída, perda da audição, déficit de memória, mobilidade e coordenação motora fina diminuídas, tremores aumentados, depressão e isolamento, recursos financeiros diminuídos relacionados com as incapacidades e outros distúrbios clínicos ${ }^{(12,13)}$.

O diagnóstico de enfermagem Ferida diabética foi identificado no membro inferior esquerdo, apresentando sinais flogísticos e fissura, recebendo atenção da equipe multiprofissional, envolvendo intervenções direcionadas ao debridamento cirúrgico e curativo diário como forma de prevenir o risco de amputação. 
Denominam-se também de "Pé diabético" e consiste em uma das complicações mais comuns do DM. São lesões nos pés de pacientes diabéticos que ocorrem em consequência de neuropatia, em $90 \%$ dos casos, doença vascular periférica e deformidades ${ }^{(13)}$. O Consenso Internacional sobre pé diabético o define como infecção, ulceração e/ou destruição de tecidos moles associadas a alterações neurológicas e vários graus de doença arterial periférica (DAP) nos membros inferiores ${ }^{(3)}$.

Como fator predisponente para o aparecimento de úlceras nos membros inferiores, é apontado à neuropatia diabética, que afeta $50 \%$ das pessoas com DM com mais de 60 anos, a qual pode estar presente antes da detecção da perda da sensibilidade protetora, resultando em maior vulnerabilidade a traumas acarretando um maior risco de ulceração(3).

Em relação às intervenções de enfermagem para o cuidado do pé diabético, ressaltam-se onze recomendações propostas pela Sociedade Brasileira de Diabetes, destacando-se às relacionadas à prevenção e ao diagnóstico, como avaliar os conhecimentos, habilidades, barreiras/ limitações para o autocuidado da pessoa com DM; assegurar que as pessoas com DM e familiares reconheçam os riscos das complicações em pés e medidas de prevenção; portadores, cuidadores e profissionais da saúde devem reconhecer a perda da sensibilidade como principal fator de risco para úlceras plantares; classificar a pessoa com DM na categoria de risco; promover alívio de pressão em região plantar; e promover o acesso ao cuidado profissional e ensino mediante as necessidades individuais e categoria de risco identificado ${ }^{(3)}$.

O diagnóstico de enfermagem Integridade da pele prejudicada esteve evidente no abdome, na região inframamária, ocasionada por infecção fúngica decorrente da condição de estar restrito ao leito e ao estado de edema que torna difícil de manter esta região limpa e seca.

As infecções na pele acometem 20\% a 50\% das pessoas com DM tipo 2 e quando estão mal controladas acontecem com uma maior ocorrência e severidade, devido à hiperglicemia causar disfunções imunológicas que o torna mais vulnerável a infecção e tenha um processo cicatricial prejudicado(3).

Em um estudo(14), este diagnóstico de enfermagem esteve presente em idosos por apresentarem alterações na pele relacionadas à elasticidade e hidratação, tornando-o mais frágil e suscetível.

Considerando-se tais agravantes que diabéticos e idosos apresentam, cuidar da pele de modo a prevenir complicações é uma prática essencial e deve fazer parte dos programas de prevenção e controle do DM, tendo como ações básicas para a manutenção da saúde da pele, hidratação, higiene, controle, vestimenta e proteção(3).

O diagnóstico de enfermagem Dor intensa em membros inferiores foi um fenômeno sempre constante no idoso diabético em estudo, agravando-se durante a noite, direcionando a assistência de enfermagem para a promoção de conforto e alívio da dor, associada à terapia medicamentosa.

A dor referida denomina-se dor claudicante e é caracterizada por dor em panturrilhas mesmo em repouso acompanhada de diminuição de pulsos periféricos, resultante do processo de obstrução aterosclerótica progressiva das artérias dos membros inferiores, característico da doença arterial obstrutiva periférica (DAOP), afetando gradualmente a qualidade de vida dos diabéti$\cos ^{(3)}$. A prevalência aumenta com a idade e que as alterações arteriais são potencializadas em pacientes idosos devido às alterações cardiovasculares fisiológicas e a diminuição no padrão de exercício/atividade que podem acentuar os fatores de risco para o desenvolvimento da $\mathrm{DAOP}^{(15,10)}$. 
Diante dos efeitos deletérios que a DAOP pode ocasionar, torna-se relevante à atenção dos profissionais de saúde para o diagnóstico precocesobre os fatores de risco (tabagismo, diabetes mellitus, hipertensão arterial, dislipidemia, agregação plaquetária, pé diabético) e modificações do perfil cardiovascular, melhorando assim, a mortalidade e a qualidade de vida destes pacientes ${ }^{(3)}$.

Acredita-se que o apoio educacional as pessoas com DM implica modificação de comportamentos, evitando-se um dos principais diagnósticos de enfermagem Falta de adesão ao regime terapêutico identificado durante a realização do estudo, responsável pela severidade das complicações crônicas instaladas no idoso diabético e no comprometimento da qualidade de vida. Esse diagnóstico de enfermagem, em idosos, favorece a internação hospitalar ou contribui para ampliar a sua duração(16).

Ao ressaltar a importância da adesão ao regime terapêutico, esta é caracterizada como adoção de comportamentos de autocuidado que inclui Atividade; Alimentação saudável; Monitorização; Medicação; Resolução de problemas; Enfrentamento saudável; e Redução de riscos; sendo a abordagem educacional do Empowerment, a responsável em promover essas mudanças de comportamentos, uma vez que se trata de uma capacitação de pacientes e familiares no manejo diário do DM, que lhes conferem autonomia e responsabilidade compartilhada com a equipe de profissionais que os assistem ${ }^{(3)}$.

O diagnóstico de enfermagem Conhecimento deficiente sobre a doença e tratamento foi evidente na admissão deste paciente diante da gravidade de complicações crônicas do DM já instaladas, sendo o mesmo um idoso jovem e o não envolvimento da família (acompanhantes) no plano terapêutico dele, fato este que surpreendeu à equipe devido à existência em nosso país de Políticas Públicas que abran- gem programas específicos voltados para tais condições crônicas como o Hiperdia para hipertensão arterial e diabetes mellitus além do Estatuto do Idoso.

Tal fato proporciona a reflexão sobre o modo como as informações nos serviços de saúde estão sendo discutidas, bem como a importância do grau de compreensão dos usuários, principalmente dos idosos, com suas limitações específicas, além de provocar a preocupação se os profissionais de saúde detêm os conhecimentos e habilidades pedagógicas capazes de oferecer ferramentas necessárias para o manejo problema de saúde com vistas ao autocuidado.

Torna-se necessário uma análise crítica dos profissionais da área de saúde, entre eles os enfermeiros, acerca de suas ações educativas para o atendimento à população que envelhece com características próprias e complexas exigindo atuação específica ${ }^{(17)}$.

Diante das dificuldades dos usuários com DM, recomenda-se a implantação de um programa de educação em diabetes, para que os usuários sejam envolvidos em todas as fases do processo educacional, visando assumir a responsabilidade do seu papel terapêutico, a partir de conhecimentos e habilidades que o instrumentalizem para o autocuidado ${ }^{(18)}$.

Com este enfoque, um programa de educação em DM seria uma alternativa dinâmica com a capacitação do indivíduo tornando-o apto a adotar novos comportamentos e desse modo, assegurar mais autonomia e independência ao idoso em busca de melhor qualidade de vida frente ao problema de saúde.

Para a Associação Americana de Educação em Diabetes, a educação em diabetes é um processo interativo, colaborativo e contínuo que envolve pessoas com DM e seu educador, que inclui a avaliação das necessidades educacionais específicas individuais; a identificação das metas individuais específicas para o au- 
togerenciamento desta doença; intervenções educacionais e comportamentais dirigidas para ajudar o indivíduo a identificar as metas do autogerenciamento; e a avaliação da capacidade dos indivíduos em atingir as metas do auto gerenciamento; configurando-se o enfermeiro como o profissional de saúde predominante nesta modalidade terapêutica(3).

\section{CONCLUSÃO}

Ao realizar este estudo de caso pode-se observar que esta pessoa idosa com DM apresentou diagnósticos de enfermagem intimamente relacionados às complicações crônicas da doença pela falta de prevenção de fatores de risco durante a sua evolução. Torna-se evidente a relevância do papel da educação em saúde ao portador de DM com foco na responsabilidade compartilhada entre equipe e o paciente para prevenção de fatores de risco e minimização de complicações decorrentes da doença. Os princípios básicos no tratamento de pacientes idosos não diferem, em geral, dos estabelecidos a indivíduos diabéticos mais jovens, entretanto, o idoso possui particularidades (déficit cognitivo e incapacidade funcional) referentes ao processo de envelhecimento que precisam ser consideradas durante a assistência de enfermagem.

Destaca-se a importância do cuidado sistematizado ao idoso portador de diabetes por proporcionar uma melhor compreensão das causas e meios que levam às complicações da doença propiciando um tratamento mais adequado às suas necessidades de saúde.

\section{REFERÊNCIAS}

1. Ministério da Saúde (Brasil). Secretaria de Vigilância em Saúde, Departamento de Análise de
Situação de Saúde. Plano de ações estratégicas para o enfrentamento das doenças crônicas não transmissíveis (DCNT) no Brasil 2011-2022. Brasília: Ministério da Saúde; 2011.

2. Lerário AC. Peculiaridades do tratamento do idoso com diabetes. Capítulo 9. In: E-book Diabetes na prática clínica. Sociedade Brasileira de Diabetes [internet] 2011 [cited 2012 Jan 20] Available from: http://www.diabetesebook.org.br

3. Sociedade Brasileira de Diabetes (Brasil). Cuidados de enfermagem em Diabetes Mellitus. Itapevi: A.C. Farmacêutica; 2011.

4. Teixeira CRS, Becker TAC, Citro R, Zanetti ML, Landim CAP.Validação de intervenções de enfermagem em pessoas com diabetes mellitus. Rev Esc Enferm USP. 2011;45(1):173-9.

5. BeckerTAC, Teixeira CRS, Zanetti ML. Diagnósticos de enfermagem em pacientes diabéticos em uso de insulina. Rev Bras Enferm. 2008;61(6): 847-52.

6. Ministério da Saúde (Brasil). Resolução n 466/12. Diretrizes e normas regulamentadoras de pesquisas envolvendo seres humanos. Diário Oficial da União 12 dec 2012; Seção 1.

7. Virgínio N. Validação de instrumento de coleta de dados de enfermagem para clientes hospitalizados [dissertação]. João Pessoa: Centro de Ciências da Saúde, Universidade Federal da Paraíba; 2003.

8. Institute for Human and Machine [homepage]. Cognition-CmapTools. University of West Florida. [Cited 2012 Mar 10] Available from: http://cmap. inmc.us

9. International Council of Nurses (Unites States of America). Classificação Internacional para a Prática de Enfermagem Versão 2011. [cited 2013 Jan 24] Available from:http://www.icn.ch/ images/stories/documents/pillars/Practice/icnp/ translations/icnp-Brazil-Portuguese_translation. pdf

10. Smeltzer SC, Bare BG, Hinke JL, Cheever KH. Brunner\&Suddarth Tratado de enfermagem médico-cirúrgica. 11. ed. Rio de Janeiro: Guanabara Koogan; 2009.

11. Borba AKOT, Marques APO, Leal MCC, Ramos RSPS. Práticas educativas em diabetes Mellitus: revisão integrativa da literatura. Rev Gaúcha Enferm. 2012;33(1):169-76.

12. Silva LMC, Palha PF, Barbosa GR, Protti ST, Ramos AS. Aposentados com diabetes tipo 2 na Saúde 
da Família em Ribeirão Preto, São Paulo - Brasil. RevEscEnferm USP. 2010;44(2):462-8.

13. Rezende KF, Nunes MAP, Melo NH, Malerbi D, Chacra AR, Ferraz MB. Internações por pé diabético: comparação entre o custo direto estimado e o desembolso do SUS. Arq Bras Endocrinol Metab. 2008;52(3):523-30.

14. Nascimento SS, Oliveira DF, Barbosa LR, Stival MM. Julgamento clínico de enfermagem em idosos portadores de Diabetes Mellitus fundamentado na teoria do déficit do autocuidado. Anuário da Produção de Iniciação Científica Discente, 2008;11(12):91-106.

15. Pereira DAG, Lustosa LP, Murta FN, Prata KO, Saraiva $\mathrm{PS}$, Cunha-Filho IT. Avaliação do equilíbrio estático em pacientes fisicamente ativos com doença arterial obstrutiva periférica - um estudo piloto. Ter Man. 2011;9(41):49-53.

16. Nóbrega OT, Karnikowski MGO. A terapia medicamentosa no idoso: cuidados na medicação. Cien Saude Colet. 2005;10(2):309-13.

17. Souza LM, Lautert L, Doll J, Silva MCS. Nursing consultation to elderly people based on andragogy: a review article. (Online)[internet]. 2009 Feb 3;[cited 2012 June 10]; 8(1). Avaliable from: http://www.objnursing.uff.br/index.php/nursing/article/view/2119

18. Oliveira KCS, Zanetti ML. Conhecimento e atitude de usuários com diabetes mellitus em um Serviço de Atenção Básica à Saúde. RevEscEnferm USP. 2011;45(4):862-8.
Todos os autores participaram das fases dessa publicação em uma ou mais etapas a seguir, de acordo com as recomendações do International Committe of Medical Journal Editors (ICMJE, 2013): (a) participação substancial na concepção ou confecção do manuscrito ou da coleta, análise ou interpretação dos dados; (b) elaboração do trabalho ou realização de revisão crítica do conteúdo intelectual; (c) aprovação da versão submetida. Todos os autores declaram para os devidos fins que são de suas responsabilidades o conteúdo relacionado a todos os aspectos do manuscrito submetido ao OBJN. Garantem que as questões relacionadas com a exatidão ou integridade de qualquer parte do artigo foram devidamente investigadas e resolvidas. Eximindo, portanto o OBJN de qualquer participação solidária em eventuais imbróglios sobre a materia em apreço. Todos os autores declaram que não possuem conflito de interesses, seja de ordem financeira ou de relacionamento, que influencie a redação e/ou interpretação dos achados. Essa declaração foi assinada digitalmente por todos os autores conforme recomendação do ICMJE, cujo modelo está disponível em http://www. objnursing.uff.br/normas/DUDE_final_13-06-2013.pdf

Recebido: $16 / 07 / 2014$

Revisado: 03/06/2016

Aprovado: 03/06/2016 